\title{
Hemorragia alveolar difusa. Serie de casos en el Hospital Universitario San Ignacio 2010-2011
}

\author{
Diffuse alveolar hemorrhage. Case series at Hospital Universitario San \\ Ignacio, 2010-2011
}

Luis Carlos Triana Moreno, MD. ${ }^{(1)}$

\section{RESUMEN}

La hemorragia alveolar difusa es un síndrome clínico, caracterizado por síntomas respiratorios asociados con anemia, junto con hallazgos, en la radiografía del tórax, de infiltrados de ocupación alveolar multilobar, además de hemoptisis (esta última no siempre presente). El abordaje diagnóstico incluye la confirmación de la hemorragia usualmente por medio de fibrobroncoscopia y determinación de la causa. La etiologia es diversa; las causas más frecuentes siguen siendo las vasculitis ANCAS positivas, el lupus eritematoso sistémico, la enfermedad de Goodpasture y la asociación con medicamentos. Se presentan los casos recogidos en los años 2010 y 2011 en el Hospital Universitario San Ignacio.

Palabras clave: hemorragia alveolar difusa, ANCAS, lupus eritematoso sistémico, capilaritis, hemosiderófagos.

\section{ABSTRACT}

Diffuse alveolar hemorrhage is a clinical syndrome characterized by respiratory symptoms associated with anemia, together with radiographic findings of multilobar alveolar occupation, besides hemoptysis (although not a constant finding). The diagnostic workup includes confirmation of hemorrhage, usually by bronchoscopy, and determination of the cause. The etiology is diverse; the most common causes continue to be the ANCA-positive vasculitides, systemic lupus erythematosus, Goodpasture disease, and drug-related. This article presents cases collected during the years 2011 and 2012 at Hospital Universitario San Ignacio.

Keywords: diffuse alveolar hemorrhage, ANCAs, systemic lupus erythematosus, capillaritis, hemosiderophages.

Rev Colomb Neumol 2012; 24: 37-40.

\section{INTRODUCCIÓN}

El síndrome de hemorragia alveolar difusa es una entidad clínica infrecuente, pero con un impacto importante sobre la morbilidad y la mortalidad (1), pues suele afectar a los individuos en edad productiva y su abordaje diagnóstico debe ser reconocido con claridad para implantar medidas terapéuticas. Aunque las causas son diversas (2), en general la mayoría de veces se circunscriben a unas pocas que incluyen vasculitis asociadas a los anticuerpos contra el citoplasma del neutrófilo (ANCAS) que son las más frecuentes (1-3), seguidas por otras como lupus eritematoso sistémico (LES), efectos secundarios de algunos medicamentos (anticoagulantes, antiagregantes) y hemosiderosis pulmonar idiopática $(1,4)$.

Durante un periodo de 21 meses, desde enero de 2010 y hasta septiembre de 2011, se recogieron los casos con diagnóstico de hemorragia alveolar difusa en el Hospital Universitario de San Ignacio. A continuación se presentan dos casos representativos.

(1) Internista, Neumólogo. Unidad de Neumología y Unidad de Cuidado Intensivo, Hospital Universitario San Ignacio, Pontificia Universidad Javeriana. Bogotá, Colombia.

Correspondencia: Ictrianam@hotmail.com

Recibido: octubre 3 de 2012. Aceptado: octubre 24 de 2012. 


\section{REPORTE DE CASOS}

\section{Caso 1}

Hombre de 36 años, arquitecto, con historia de enfermedad renal crónica de etiología desconocida desde hace nueve años, en manejo con diálisis peritoneal. Consultó por cuadro de un mes de evolución de disnea progresiva, tos seca, hiporexia, astenia y adinamia. Fue valorado por Neumología por la presencia de una lesión nodular en el pulmón derecho; se realizó biopsia guiada por tomografía computarizada (TAC) con patología compatible con neumonia en organización, razón por la cual recibió esteroides sistémicos, con resolución parcial de los síntomas.

Diez meses después presentó tos hemoptoica, deterioro de la clase funcional, hiporexia y fiebre no cuantificada. La tomografia de tórax de alta resolución (TACAR) mostró nódulos en vidrio esmerilado y parches alveolares diseminados en ambos campos pulmonares (Figura 1); se realizó fibrobroncoscopia con lavado broncoalveolar con líquido de características hemorrágicas y en el estudio citológico con hemosiderófagos mayores al $80 \%$, hallazgos compatibles con hemorragia alveolar. Dado el diagnóstico previo y el cuadro actual, se realizó biopsia pulmonar abierta, la cual mostró hemosiderófagos, capilaritis y necrosis en los vasos (Figura 2).

En vista de los hallazgos y teniendo en cuenta una lesión renal establecida que fue aguda y de causa que indeterminada, se sospechó vasculitis de pequeños vasos, por lo que se tomaron c-ANCAS los cuales fueron positivos en una dilución de $1 / 20$. Al confirmar el diagnóstico de vasculitis pulmonar asociada a c-ANCAS, se inició tratamiento con bolos de metilprednisolona y

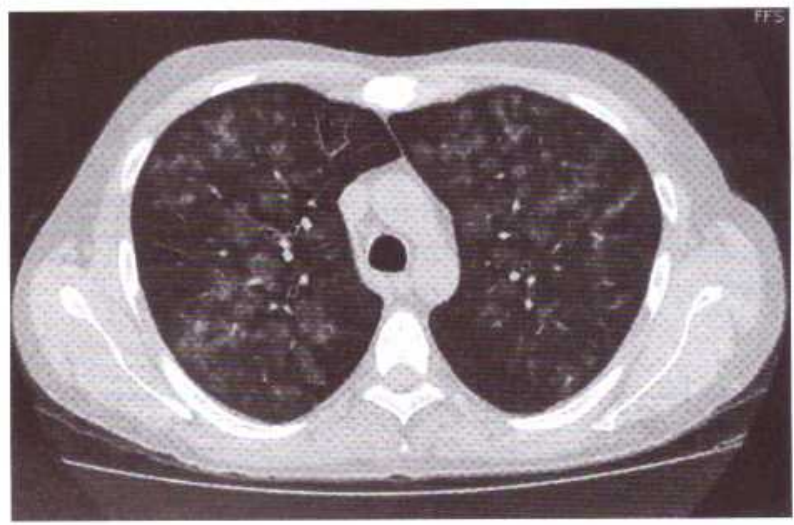

Figura 1. Corte sagital de TACAR de tórax con parches de ocupación alveolar y nódulos en vidrio esmerilado de distribución difusa. posteriormente dosis decrecientes de corticoides sistémicos y azatioprina, con mejoría del cuadro clínico y de los hallazgos radiológicos. Sin embargo, el paciente falleció un año después del diagnóstico por choque séptico refractario.

\section{Caso 2}

Paciente masculino de 28 años, natural y procedente de Puente Nacional, Santander. Con antecedente de LES diagnosticado desde los 17 años de edad, sindrome antifosfolipido asociado y nefropatía lúpica estadio IV, con múltiples recaídas y mala respuesta al tratamiento con corticoides, ciclofosfamida y rituximab.

Consultó por cuadro de una semana de evolución de disnea progresiva hasta el reposo, sin hemoptisis y tos seca persistente; al exam en físico tenía palidez marcada, polipnea, taquicardia y estertores diseminados en ambos campos pulmonares. La TACAR de ingreso mostró infiltrados alveolares y vidrio esmerilado multilobares (Figura 3) y el hemograma anemia macrocítica.

Con sospecha de hemorragia alveolar se realizó fibrobroncoscopia, la cual mostró sangrado activo de todos los segmentos y retorno de líquido de características hemorrágicas; en la citología se observaron hemosiderófagos del $100 \%$.

Con diagnóstico de hemorragia alveolar difusa asociada a LES, se administraron bolos de metilprednisolona y ciclofosfamida. El paciente progresó rápido a falla respiratoria hipoxémica, que requirió intubación orotraqueal y soporte ventilatorio; por la severidad del cuadro clínico se realizó plasmaféresis. Falleció por hemorragia alveolar refractaria y choque séptico de origen pulmonar.

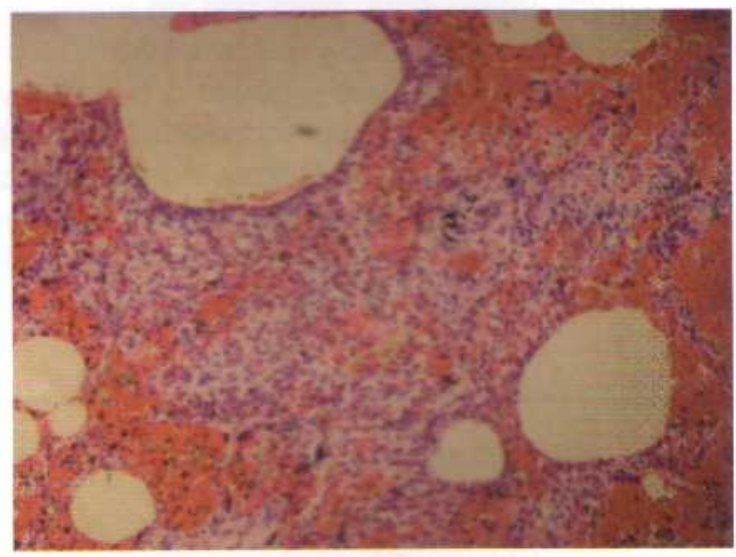

Figura 2. Biopsia abierta de pulmón, con alvéolos ocupados por glóbulos rojos, hemosiderófagos y capilaritis. 
Durante los años 2010 y 2011 en el Hospital Universitario San Ignacio, se recolectaron en total siete casos de hemorragia alveolar difusa de etiología diversa (Tabla 1).

\section{DISCUSIÓN}

El sindrome de hemorragia alveolar difusa, es una entidad infrecuente; sin embargo, dado el impacto en términos de morbimortalidad ha merecido la atención de varios grupos en la literatura médica (5-7).

En un estudio de cohorte retrospectivo reciente con más de 100 pacientes inmunocompetentes realizado en Francia (8), $48 \%$ de las causas fueron agrupadas como de etiología inmune, siendo las más frecuentes las relacionadas con vasculitis sistémicas asociadas a ANCAS y LES. Dentro de las causas no inmunes la más común fue la asociada a falla cardiaca congestiva, seguida por las de etiología infecciosa, barotrauma e

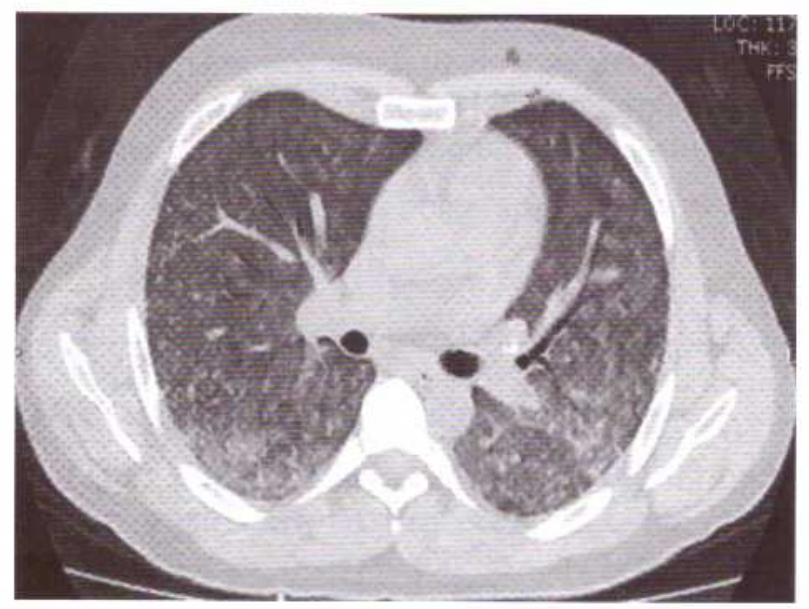

Figura 3. Corte sagital de TACAR de tórax a nivel de lóbulos inferiores con vidrio esmerilado y ocupación alveolar difusa. idiopáticas. Dicha revisión confirma lo que otras series $(6,7)$ han mostrado, y es que la tríada clásica de presentación clínica de hemoptisis, anemia e infiltrados de ocupación alveolar en la radiografía del tórax está presente en el momento de la consulta en alrededor del $40 \%$ de los casos y la hemoptisis está ausente en un tercio de los pacientes $(8,9)$.

Diagnosticar hemorragia alveolar difusa y aclarar su etiología es un reto clínico; su mortalidad es particularmente alta en los casos asociados a lupus eritematoso sistémico (aproximadamente $50 \%)(8,10)$, falla cardiaca congestiva $(8,9)$ y vasculitis asociada a ANCAS $(7,8,11)$.

Iniciando por la sospecha clínica de hemorragia alveolar, el primer paso diagnóstico para confirmarla debe ser la realización de la fibrobroncoscopia la cual muestra la presencia de sangrado activo proveniente de diferentes luces bronquiales, y un lavado broncoalveolar que es de color rojo franco o rosado. En caso de ausencia de estos hallazgos, el estudio citológico del lavado broncoalveolar con tinción de azul de Prusia, documenta los macrófagos cargados de hemosiderina, que cuando están presentes en un porcentaje mayor al $20 \%$, indican una hemorragia alveolar $(5,6,8,12)$.

Una vez confirmada la hemorragia, la búsqueda etiológica dependerá de las características clínicas asociadas, como compromiso de otros sistemas, en especial renal, importante para el diagnóstico de una causa inmune, los signos clínicos y ecocardiográficos para la falla cardiaca y los marcadores de inflamación aguda para los procesos infecciosos $(8,12)$.

Los estudios serológicos son importantes cuando la sospecha de etiología inmune es alta. En este sentido es fundamental el estudio por inmunofluorescencia de los ANCAS ( $p$ y $c$ ) y los anticuerpos antinucleares (ANAS $y$ anti DNA) en caso de que el diagnóstico de LES aún no se haya realizado $(8,9,12)$.

Tabla 1. Características de los casos de hemorragia alveolar.

\begin{tabular}{|llcllll|}
\hline Caso & Sexo & Edad & \multicolumn{1}{c}{ Causa } & Nefropatía & Biopsia pulmonar & Aféresis \\
\hline 1 & Masculino & 36 & C-ANCAS & En diálisis & Capilaritis & No \\
2 & Masculino & 28 & LES & GN en diálisis & No & Si \\
3 & Femenino & 54 & p-ANCAS & GN en diálisis & No & Si \\
4 & Femenino & 64 & Crioglobulinemia & GNRP & No & Si \\
5 & Masculino & 41 & Hemosiderosis & Postrasplante & Si & No \\
6 & Femenino & 21 & LES & GN & No & Si \\
7 & Femenino & 70 & p-ANCAS & GN & No & Si \\
\hline
\end{tabular}

GN: glomerulonefritis; GNRP: glomerulonefritis rápidamente progresiva 